\title{
Stump Appendicitis
}

\author{
Filip Bednar, James Skiba, ${ }^{2}$ Ali F. Mallat, and Lena M. Napolitano
}

A 53-YEAR-OLD female presented to the emergency department with abdominal pain localized to the right lower quadrant associated with intermittent episodes of nausea and emesis. Her past surgical history was notable for laparoscopic appendectomy 20 mo prior for acute appendicitis. The abdominal CT scan obtained at that time identified a right renal mass that was confirmed to be renal cell carcinoma, and she underwent robotic right partial nephrectomy 2 mo after appendectomy. Her past medical history included hypertension treated with lisinopril and hydrochlorothiazide. Physical examination demonstrated temperature $36.1^{\circ} \mathrm{C}$, heart rate 72 beats/min, respiratory rate 16 breaths/min, blood pressure $120 / 69 \mathrm{mmHg}, \mathrm{SpO}_{2} 95 \%$ on room air. Abdominal examination confirmed point tenderness in the right lower quadrant with no guarding or rebound. Laboratory studies revealed a leukocytosis of $12.9 \times 10^{9} / \mathrm{L}$ with a predominant neutrophilia of $75.9 \%$. All other studies were normal.

A CT scan of the abdomen and pelvis with enteral and intravenous contrast (Fig. 1) demonstrated evidence of acute stump appendicitis with a 4-cm appendiceal remnant with radiopaque suture material at the tip. The stump was fluidfilled and dilated up to $1.5 \mathrm{~cm}$ with adjacent inflammatory change. Systemic antibiotics were initiated and she was admitted. Her abdominal pain improved, and she was discharged home on a course of oral antibiotics. Elective laparoscopic stump appendectomy was performed, and surgical pathology confirmed a $3-\mathrm{cm}$ long, 1-cm diameter distal segment of appendix that was confirmed to be the appendiceal stump with no notable abnormality.

Stump appendicitis is a rare entity and an uncommon late complication of appendectomy, where inflammation occurs in the remaining appendiceal stump [1]. Stump appendicitis requires early detection and diagnosis. Typically, patients present with signs and symptoms similar to acute appendicitis; however, due to prior surgery, the diagnosis is difficult and the rate of appendiceal stump perforation is high [2].

A recent systematic review identified 60 published cases of stump appendicitis, and documented that the interval from the original appendectomy ranged from $4 \mathrm{~d}$ to $50 \mathrm{y}$, and that perforation with gangrene of the stump occurred in $40 \%$ of cases [3]. A high level of suspicion of stump appendicitis is required in patients presenting with right lower quadrant abdominal pain and a surgical history of appendectomy, and ultrasound or computed tomography abdominal imaging is

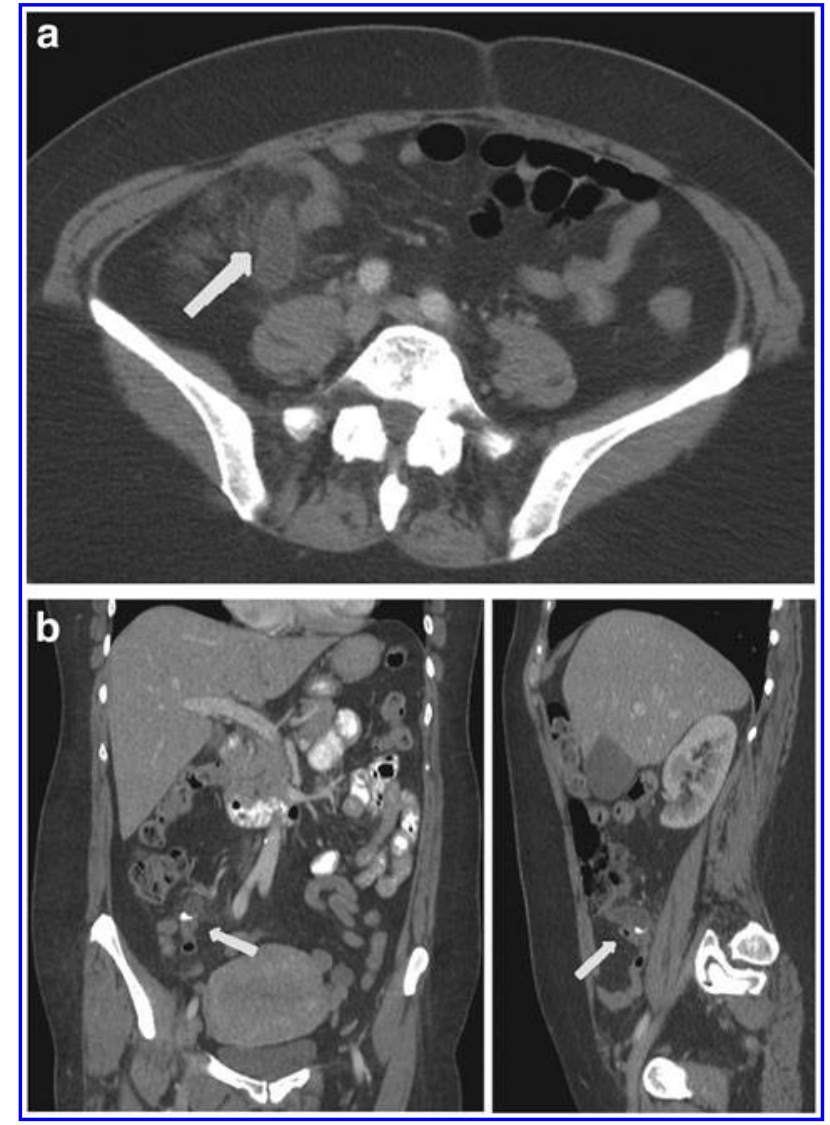

FIG. 1. Computed tomography of abdomen, axial (a) and saggital/coronal reconstructed sections (b). Demonstration of a $4-\mathrm{cm}$ appendiceal remnant with radiopaque suture material at its tip. This appendiceal stump is fluid-filled and dilated up to $1.5 \mathrm{~cm}$ with adjacent inflammatory changes, but no associated perforation or abscess. There are post-operative changes associated with the right partial nephrectomy.

diagnostic in most cases. The main cause of stump appendicitis is the persistence of a large appendicular remnant. Treatment consists of a completion appendectomy by either a laparoscopic or open approach.

Correct identification and removal of the appendix at its base without leaving an appendiceal stump at initial

\footnotetext{
${ }^{1}$ Division of Acute Care Surgery (Trauma, Burn, Surgical Critical Care, Emergency Surgery), Department of Surgery, ${ }^{2}$ Department of Emergency Medicine, University of Michigan Hospital and Health Systems, Ann Arbor, Michigan.
} 
appendectomy minimizes the risk of stump appendicitis [4]. To prevent stump appendicitis, some have proposed use of the "critical view" similar to that used in laparoscopic cholecystectomy. The "critical view" ensures proper identification of the entire appendix and its base, the appendix retracted to the left in the 10 o' clock position, visualization of the taenia libera in the 3 o'clock position, and confirmation of the terminal ileum at the 6 o'clock position, with transection of the appendiceal base to allow $<0.5 \mathrm{~cm}$ appendiceal stump [5].

\section{References}

1. Kanona H, Al Samaraee A, Nice C, et al. Stump appendicitis: a review. Int J Surg. 2012;10(9):425-428.

2. Liang MK, Lo HG, Marks JL. Stump appendicitis: A comprehensive review of literature. Am Surg 2006;72:162166.
3. Leff DR, Sait MR, Hanief M, et al. Inflammation of the residual appendix stump: a systematic review. Colorectal Dis. 2012;14:282-293.

4. Roberts KE, Starker LF, Duffy AJ, et al. Stump appendicitis: a surgeon's dilemma. JSLS. 2011;15:373-378.

5. Subramanian A, Liang MK. A 60-year literature review of stump appendicitis: the need for a critical view. Am J Surg 2012;203:503-507.

Address correspondence to: Dr. Lena M. Napolitano Division of Acute Care Surgery

(Trauma, Burn, Surgical Critical Care, Emergency Surgery) Department of Surgery University of Michigan Hospital and Health Systems 1500 E. Medical Center Dr., UH 1C-340 Ann Arbor, MI 48109-5033

E-mail: lenan@umich.edu 